\title{
ELABORACIÓN. ANÁLISIS DE CONFIABILIDAD Y VALIDEZ DEL TEST DE PERSPECTIVA
}

\author{
Angel Guevara Pino
}

\begin{abstract}
RESUMEN
El presente estudio se basa en las implicaciones que tiene la perspectiva en la organización del espacio y el desarrollo del proceso cognitivo. Se trabajó con una muestra total de 973 estudiantes que cursan entre el 4to - 6to de Primaria y 1 ro - 2do de Secundaria pertenecientes a Centros Educativos Estatales y Particulares de la ciudad de Lima. Queremos despejar las siguientes interrogantes: ¿ Conocer el valor de la confiabilidad y de la validez del Test de Perspectiva? y también ¿Señalar si la edad cronológica, el género y el tipo de centro educativo presentan diferencias significativas en el rendimiento del Test de Perspectiva? Se determinó la confiabilidad con el Método de Consistencia Interna y el Método de las Mitades encontrando coeficientes de correlación altamente significativos, lo que significa que el test presenta una Alta constancia y precisión de medición. La Validez Concurrente se obtuvo correlacionando la Batería de Aptitudes Diferenciales y Generales - Nivel Elemental (BADYG-E) con el Test de Perspectiva encontrando coeficientes significativos, siendo mejor el coeficiente de correlación con el factor no verbal (I.G.Nv) con los sub-tests de Razonamiento Lógico y Aptitud Espacial; mientras que para la Validez de Constructo se utilizó el Análisis Factorial correlacionando las variables examinadas, encontrando tres factores mediante la rotación Varimax, los cuales dependen de las dimensiones predominantes en las figuras en la presencia de dos o más elementos de las figuras y en el dominio del volumen en las figuras. Al concluir el análisis hemos obtenido un instrumento válido y confiable, donde se encontró que hay diferencias significativas en la perspectiva por edad cronológica, por genero y por el tipo de centro educativo Finalmente se presentan tablas por períodos de edad, por genero y por tipo de colegio en donde los puntajes directos fueron transformados a puntuaciones estándar normalizadas T para la interpretación de los puntajes del Test de Perspectiva.
\end{abstract}

Palabras Claves: Plano, Perspectiva, Espacio Proyectivo, Imagen Mental.

\begin{abstract}
The present study is based on the implications that hel she has the perspective in the organization of the space and the development of the process cognitive. One worked ll'ith a total sample of 973 students that your they study among the 4to - 6to of Primary and 1ro 2do of Secondary belonging to State Educational Centers and Peculiar of the city of Lima. We want to elear the following queries: To know the value of the dependability and of the validity of the Test of Perspective? and also to Point out if the chronological age, does the one generate and does the type of educational center present significant differences in the yield of the Test of Perspective? was the dependability determined with the Method of internal Consistency and the Method of the Halves finding highly significant correlation coefficients, what means that the test presents a High perseverance and menstruation precision. The Concurrent Validity was obtained correlating the Battery of Differential and General Aptitudes-Elementary Level (BADYG-AND) with the Test of Perspective finding significant coefficients, being better the correlation coefficient with the non verbal factor (1.G.Nv) with those sub-tests of Logical Reasoning and Space Aptitude; while for the Validity of construct the Factorial Analysis was used correlating the examined variables, finding three factors by means of the rotation Varimax, which depend on the predominant dimensions in the figures in the presence of two 01' more elements of the figures and in the domain of the volume in the figures. When concluding the analysis we have obtained an instrument been worth and reliable, where it was found that there are significant differences in the perspective for chronological age, for I generate and for the type of educational center charts are presented finally by periods of age. for I generate and for type of school where the direct punctuation was transformed to punctuation normalized standard $T$ for the interpretation of the punctuation of the Test of Perspective.
\end{abstract}

Key words: Plane, Perspective, Space Projective, Mental image. 
En el desarrollo de su teoría sobre la naturaleza del pensamiento y el razonamiento de los niños, Piaget descubrió en sus investigaciones que el niño normal atraviesa cuatro estadíos principales en su desarrollo cognitivo: el sensorio-motor, el preoperatorio, el de las operaciones concretas y el de las operaciones formales.

Durante el período de las operaciones concretas, los niños examinados por Piaget llegaron a la plena comprensión de los conceptos topológicos, en tanto que los conceptos referentes a la proyección, tales como los de perspectiva y de secciones, se iban dominando gradualmente (Piaget e Inhelder, 1956).

El espacio proyectivo o geometría proyectiva es el tipo de espacio en el cual está centrada nuestra investigación, el propósito es rastrear la conciencia evolutiva del niño de su propio punto de vista y su relación con otros, es la de las relaciones perspectivas en relación con un punto de vista, es decir, la coordinación de perspectivas aspecto que fue explorado por Piaget mediante el "Modelo de las Tres Montañas". Durante los desplazamientos, únicamente se conservan las rectas y ciertas relaciones cuantitativas, pero no las paralelas, los ángulos o las distancias.

Basándonos en estos estudios que a su vez están interrelacionados con la anticipación y la organización del espacio, que son componentes en el desarrollo de la imagen mental y el proceso cognitivo; esta investigación tiene como propósito elaborar un instrumento orientado a medir este complejo proceso psíquico teniendo los siguientes objetivos:

- ¿Conocer el valor de la confiabilidad y de la validez del Test de Perspectiva?

- ¿Señalar si la edad cronológica, el género y el tipo de centro educativo presentan diferencias significativas en el rendimiento del Test de Perspectiva?

La Funcionalidad Psicológica de la Perspectiva se explica en el desarrollo mental del niño, pues este aparece como una construcción progresiva tal que cada innovación sólo se hace posible en función de la anterior. Siguiendo el principio de Piaget, el niño durante el estadío operacional concreto desarrolla la perspectiva que es la capacidad para ver las cosas a partir de más de un punto de vista (Coordinación de Perspectivas). Al pasar el niño al estadío operacional formal puede pensar acerca de las posibilidades y une estos temas mediante la capacidad para pensar no sólo en los hechos reales sino imaginarse también como serían (Marcos de Referencias Hipotéticos).

En base a esto se puede considerar al estadío operacional concreto como un tiempo de perfeccionamiento de las capacidades individuales y al estadío operacional formal como un tiempo de ampliar estas capacidades y de organizarlas en estrategias mentales eficientes que forman parte finalmente de la Lógica Deductiva.

Beard (1968) encontró que se puede desarrollar la perspectiva mediante la estimulación de experiencias espaciales en el niño, como la vista de fotografías aéreas en la televisión, el uso de juguetes de construcción simples, con los que se hacen modelos y el estudio de los diagramas y planos que acompañan a algunos de esos juguetes.

La importancia en la exploración, estimulación y desarrollo de la perspectiva, se justifica porque en la actualidad forma parte de los tests de aptitud, en los sub-tests que evalúan el factor espacial (que se traducen en la mayor o menor aptitud para manipular mentalmente objetos geométricos) por ejemplo la Batería de Aptitudes Diferenciales y Generales - el DAT entre otros test de aptitud, que son útiles para realizar una adecuada orientación vocacional, tomando en cuenta el factor espacial para las carreras como arquitectura, diseño 
gráfico, la decoración, las artes plásticas, el dibujo industrial, etc.

\section{CARACTERISTICAS GENERALES DEL TEST DE PERSPECTIVA}

\section{Ficha Técnica}

Nombre : : Test de Perspectiva.

Autor : Ps. Ángel Guevara.

Procedencia : Lima (Perú).

Aplicación : Individual y Colectiva.

Ámbito de Aplicación : De 9 a 15 años de edad.

Tiempo de Aplicación : Aproximadamente 45 minutos.

Corrección : Manual

Puntuación : Número total de aciertos (Un punto por ítem).

Significación : Evalúa la perspectiva mediante las vistas o Planos Principales de Proyección (Frontal, de Perfil y Horizontal) en figuras de diferentes objetos tridimensionales

\section{DESCRIPCIÓN DE LA PRUEBA}

La prueba está compuesta por 21 figuras de objetos tridimensionales cuyo nombre está ubicado en la parte superior, el objeto que se encuentra a la izquierda sirve de patrón, mientras que las alternativas son los recuadros de las vistas o proyecciones del objeto que se encuentran a la derecha con su respectiva letra en la parte superior (A, B, C y D) y donde sólo una de ellas es la vista correcta del objeto.

Los 21 objetos tendrán sus 4 recuadros respectivos a su derecha, que son las vistas de como se deberían ver a los objetos en un plano de proyección determinado (Frontal, de Perfil o Horizontal), tres de las vistas han sido modificadas o alteradas y una de estas tres ha sido duplicada quedando de esta manera sólo una vista correcta la cual debe ser señalada por el examinado.

El orden de los objetos así como el de las alternativas ha sido distribuido al azar, teniendo a las dos primeras figuras como ejemplos que no son contabilizados en el puntaje total del test.

Las figuras de objetos conocidos que se emplean son las siguientes: Cruz y Hacha (ejemplos) Escritorio - Mesa - Semáforo Candado - Borrador - Archivo - Silla Tranquera Libro - Regla - Sello - Cerca - Interruptor - Maletín - Jarra - Botiquín- Arco - Martillo Tacho - Refrigerador Señal

\section{Método de Administración de los Items}

\section{A) Consideraciones para la Administración}

El psicólogo o evaluador mientras distribuye los cuadernillos y las hojas de respuesta debe decir: "Guarden todas sus cosas sólo necesitarán un lápiz o lapicero", "No abran el cuadernillo antes de que se les diga que lo hagan", luego se realiza una explicación general del objetivo de la evaluación motivando a los estudiantes para que trabajen en silencio y de forma individual, finalmente se empieza se inicia la prueba leyendo en voz alta las instrucciones generales y específicas. 


\section{B) Instrucciones Generales}

En primer lugar se llenan los datos personales que figuran en la hoja de respuestas y a continuación se da la siguiente indicación señalada en la Hoja de Respuestas: En la vida diaria nosotros vemos objetos unas veces viéndolos desde arriba, otras veces de frente y otras desde un lado o costado.

Te indicamos que sólo una de las 4 figuras que acompañan al objeto representa la vista correcta del objeto.

Trabaja como en los siguientes ejemplos:

¿Cuál es la forma correcta en que se está viendo la cruz?

¿Cuál es la forma correcta en que se está viendo el hacha?

Marca con un aspa $(X)$ la alternativa correcta que creas es la correcta en la hoja de respuestas de la prueba.

\section{C) Instrucciones Específicas}

Para que el estudiante comprenda de manera practica la consigna, el examinador puede utilizar como ejemplo la pizarra del salón de la siguiente manera: de que manera están ustedes viendo la pizarra del salón (de frente), de que manera la estoy viendo - el examinador observa desde un lado la pizarra (de costado) y si tuviéramos una escalera como veríamos la pizarra (desde arriba).

Trabajamos igualmente con los ejemplos de la prueba (Cruz y Hacha): observen la figura y ahora vean en la alternativa A ¿de qué manera se está viendo la cruz? y así sucesivamente mencionando las cuatro alternativas del ítem o de la figura.

Seguidamente se les indica que en la hoja de respuestas sólo deben marcar una alternativa en cada figura, teniendo mucho cuidado en marcar más de una sola alternativa o recuadro.

Finalmente se les pregunta a los estudiantes si entendieron la manera como tienen que desarrollar la prueba o si tienen alguna duda para resolverla en el momento y dar inició a la evaluación, indicándoles que tienen 30 minutos para desarrollar la prueba.

\section{CORRECCIÓN Y CALIFICACIÓN}

Se corrige a través de las claves de respuestas: 1 punto por ítem contestado correctamente y O si el estudiante lo hacía incorrectamente (discrepaba) marcaba dos veces o no marcaba, seguidamente en el protocolo son sumados los puntajes obteniendo el Puntaje Total que luego es ubicado en la tabla donde están señaladas las Categorías de Rendimiento del test.

\section{MÉTODO}

\section{Muestra}

Estuvo compuesta por 973 estudiantes pertenecientes a diferentes centros educativos 2 estatales y 3 particulares distribuidos según las variables de estudio (Ver Cuadro $\mathrm{N}^{\circ} 1$ ).

\section{ANÁLISIS DE LOS RESULTADOS \\ Análisis de Ítems}

Los ítems del presente test han sido analizados en función al índice de dificultad y a la correlación tetracórica con un total de 973 estudiantes. Por el índice de dificultad se selecciono los ítems comprendidos entre 0.40 y 0.60 de dificultad, los Ítems iguales o menores que 0.39 e iguales o mayores que 0.61 fueron eliminados.

La correlación tetracórica se efectúo con el propósito de calcular el coeficiente de correlación de las respuestas correctamente dadas correspondientes al grupo superior en contraposición del grupo inferior. El coeficiente de resultar significativo y con dirección 
positiva indica que el ítem discrimina adecuadamente. A continuación presentamos los resultados encontrados:

\section{Cuadro $\mathbf{N}^{0} 1$}

Distribución compuesta de la Muestra por Variables y Categorías

\begin{tabular}{|c|c|c|c|c|c|c|}
\hline \multicolumn{3}{|c|}{ Edad } & \multicolumn{2}{|c|}{ Género } & \multirow{3}{*}{$\begin{array}{c}\text { Por } \\
\text { edad } \\
21 \\
101 \\
140 \\
108 \\
142 \\
118 \\
36 \\
21\end{array}$} & \multirow{2}{*}{$\begin{array}{l}\text { Por } \\
\text { C.E. }\end{array}$} \\
\hline & & & V & $\mathbf{M}$ & & \\
\hline \multirow{2}{*}{$\begin{array}{l}0 \\
z \\
0 \\
0 \\
0 \\
0 \\
0 \\
0 \\
0 \\
0 \\
0 \\
0 \\
0\end{array}$} & 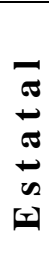 & $\begin{array}{c}8 \\
9 \\
10 \\
11 \\
12 \\
13 \\
14 \\
15\end{array}$ & & $\begin{array}{c}10 \\
51 \\
74 \\
56 \\
74 \\
50 \\
15 \\
5\end{array}$ & & 687 \\
\hline & 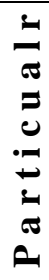 & $\begin{array}{c}8 \\
9 \\
10 \\
11 \\
12 \\
13 \\
14 \\
15\end{array}$ & & $\begin{array}{c}0 \\
9 \\
26 \\
33 \\
36 \\
15 \\
6 \\
0\end{array}$ & $\begin{array}{c}2 \\
27 \\
54 \\
73 \\
75 \\
43 \\
12 \\
0\end{array}$ & 286 \\
\hline & Sexo & 513 & 460 & & \\
\hline
\end{tabular}

Tabla $\mathbf{N}^{\circ} \mathbf{1}$

Análisis de ítems del Test de Perspectiva en una muestra de 973 estudiantes

\begin{tabular}{|l|c|c|c|}
\hline \multicolumn{1}{|c|}{ Ítems } & $\begin{array}{c}\text { Índice } \\
\text { De Dif. }\end{array}$ & $\begin{array}{c}\text { Discr. } \\
\text { Tetrac. }\end{array}$ & Decisión \\
\hline Escritorio & 0.80 & 0.50 & Anulado \\
Mesa & 0.75 & 0.40 & Anulado \\
Semáforo & 0.51 & 0.70 & Aceptable \\
Candado & 0.28 & 0.30 & Anulado \\
Borrador & 0.43 & 0.80 & Aceptable \\
Archivo & 0.56 & 0.90 & Aceptable \\
Silla & 0.51 & 0.70 & Aceptable \\
Tranquera & 0.42 & 0.90 & Aceptable \\
Libro & 0.54 & 0.70 & Aceptable \\
Regla & 0.57 & 0.80 & Aceptable \\
Sello & 0.24 & 0.30 & Anulado \\
Cerca & 0.46 & 0.80 & Aceptable \\
Interruptor & 0.54 & 0.70 & Aceptable \\
Maletín & 0.59 & 0.70 & Aceptable \\
Jarra & 0.44 & 0.80 & Aceptable \\
Botiquín & 0.52 & 0.70 & Aceptable \\
Arco & 0.45 & 0.70 & Aceptable \\
Martillo & 0.55 & 0.80 & Aceptable \\
Tacho & 0.46 & 0.70 & Aceptable \\
Refrigerador & 0.59 & 0.60 & Aceptable \\
Señal & 0.44 & 0.70 & Aceptable \\
\hline
\end{tabular}

Por este análisis del total de ítems que son 21, se descartaron 4 ítems y se han retenido 17 ítems que deben ser ordenados según este análisis.

\section{CONFIABILIDAD}

La precisión del test para medir la perspectiva esta dado por el error de medición del test encontrado con el Método de Consistencia Interna y el Método de las Mitades. 


\section{Método de Consistencia Interna}

Para este método se utilizó la formula de Kuder-Richardson para el total de la prueba, ya que no se distinguen subtest en la prueba general, no obstante hemos creído conveniente calculado para estudiantes de colegios estatales, particulares y para la muestra total.

Tabla $\mathbf{N}^{\circ} 2$

Coeficientes de consistencia interna según el tipo de colegio y la muestra total en los resultados del Test de Perspectiva

\begin{tabular}{|c|c|c|}
\hline Tipo de Colegio & $\mathbf{r ~ 2 0}$ & Decisión \\
\hline Estatales & 0.85 & Confiable \\
Particulares & 0.88 & Confiable \\
\hline Total & $\mathbf{0 . 8 7}$ & Confiable \\
\hline
\end{tabular}

Como se puede observar en todos los casos los coeficientes hallados son mayores que 0.80 lo que deja entre ver, que la variabilidad de cada uno de los ítems respecto a la variabilidad del puntaje total corresponde a una misma unidad de medida con bajo margen de error.

\section{Método de las Mitades}

Para este análisis se obtuvo las correlaciones de las puntuaciones pares e impares, tanto para los estudiantes de colegios estatales, particulares y la muestra en general; los resulta dos de ambas mitades fueron correlacionadas con el Coeficiente de Correlación de Pearson y corregidos con la formula profética de Spearman Brown, en estos resultados se podrá además ver el índice de fiabilidad y el error de medición, en la tabla que a continuación presentamos:

\section{Tabla $\mathbf{N}^{0} 3$}

Confiabilidad por el Método de las Mitades según el Tipo de Colegio y la Muestra Total en el Test de Perspectiva.

\begin{tabular}{|l|c|l|l|c|}
\hline $\begin{array}{l}\text { Tipo de } \\
\text { Colegio }\end{array}$ & $\begin{array}{l}\text { Corrl. por Mit. } \\
\text { Pun. Par-Impar }\end{array}$ & $\mathbf{R}$ & $\begin{array}{l}\mathbf{I F} \\
(\text { Rox })\end{array}$ & $\begin{array}{c}\text { Error } \\
\text { Medc. }\end{array}$ \\
\hline Estatales & 0.73 & 0.85 & 0.92 & 0.08 \\
Particulares & 0.81 & 0.89 & 0.95 & 0.05 \\
\hline Totales & 0.77 & 0.87 & 0.93 & 0.07 \\
\hline
\end{tabular}

De estos resultados se concluye que la prueba tiene una alta confiabilidad ya que sus errores de medición van desde un mínimo de 0.05 hasta un máximo de 0.08 , en conclusión nos encontramos ante un test con alta con fiabilidad.

Con el propósito de analizar el comportamiento de cada una de las mitades con el puntaje total, hemos efectuado un análisis complementario correspondiente a la mitad de los ítems impares, los ítems pares y la prueba total, que a continuación se reproduce:

Tabla $\mathbf{N}^{\circ} 4$

Coeficientes de correlación de las mitades del test con la Prueba Total

VALIDEZ

\begin{tabular}{|c|c|c|c|}
\hline Correlación & Impar & Par & Total \\
\hline Impar & 1 & 0.77 & 0.94 \\
Par & 0.77 & 1 & 0.94 \\
\hline
\end{tabular}

Se busca averiguar si el test mide lo que queremos que mida, es decir, si mide en nuestro caso la perspectiva, la validez del test se encontró mediante los Métodos de Validez Concurrente y Validez de Constructo. 


\section{Validez Concurrente}

En este método partimos del supuesto que una variable " $\mathrm{x}$ " si es interdependiente con una variable "y" como un elemento subyacente debe encontrarse correlación significativa. Para este fin la variable seleccionada ha sido la Madurez Intelectual con la Batería de Aptitudes Diferenciales y Generales - Elemental (B.A.D.Y.G-E) y sus componentes correlacionándolos con el Test de Perspectiva, que es el test materia de la presente tesis; las puntuaciones de ambos test y sus componentes se correlacionaron con el propósito de averiguar si existe o no interdependencia o concurrencia.

En la Batería de Aptitudes Diferenciales y Generales - Elemental (B.A.D.Y.G E), la confiabilidad se obtuvo mediante el Método de las Dos Mitades obteniendo en los factores globales (M.I - LG.V - LG.Nv) coeficientes que van de 0.95 a 0.90 , mientras que en los factores primarios (H.M.V - Ap.N -C.V - H.M.Nv - R.L - Ap.E) se encontraron coeficientes que van de 0.81 a 0.59 . La validez se obtiene correlacionando esta batería con otros tests (Test de Arthur S. Otis y Roger T. Lennon - Test de Flanagan - T.H.E Edición del SCAT - Test de Comprensión Yerbal - Raven) y con calificaciones escolares encontrando como resultados coeficientes que van de 0.83 a 0.55 .

Al efectuar las correlaciones entre el Test de Perspectiva y la Batería de Aptitudes Diferenciales y Generales-Elemental (B.A.D.Y.G-

E), encontramos coeficientes que van desde un mínimo de 0.14 hasta un máximo de 0.34 , resultados que pueden verse en la siguiente tabla:

\section{Tabla $\mathbf{N}^{\circ} 5$}

\section{Coeficientes de Correlación entre las puntuaciones del B.A.D.Y.G-E con el Test de Perspectiva.}

\begin{tabular}{|l|l|l|l|}
\hline \multicolumn{1}{|c|}{ Correlaciones } & r & t & Decisión \\
\hline Madurez Intelectual - Test de Perspectiva & 0.34 & 11.27 & Significativa \\
Intelig. Gral. Yerbal- Test de Perspectiva & 0.24 & 7.70 & Significativa \\
Intelig. Gral. No Yerbal- Test de Perspectiva & 0.36 & 12.02 & Significativa \\
Hab. Mental Yerbal - Test de Perspectiva & 0.14 & 4.41 & Significativa \\
Aptitud Numérica - Test de Perspectiva & 0.21 & 6.69 & Significativa \\
Comprensión Yerbal - Test de Perspectiva & 0.26 & 8.39 & Significativa \\
Hab. Mental No Yerbal - Test de Perspectiva & 0.24 & 7.70 & Significativa \\
Razonamiento Lógico - Test de Perspectiva & 0.32 & 10.52 & Significativa \\
Aptitud Espacial - Test de Perspectiva & 0.33 & 10.89 & Significativa \\
\hline
\end{tabular}

De la tabla se desprende que de los 9 coeficientes de correlación calculados el 100\% de las correlaciones son significativas al 0.05 de margen de error, lo que quiere decir que la Madurez Intelectual medida por el B.A.D.Y.G-E le subyace la Perspectiva y viceversa siendo mejor el coeficiente de correlación con el factor no verbal (LG.Nv) con los sub-tests de Razonamiento Lógico y Aptitud Espacial. Por lo hallado se concluye que el Test de Perspectiva tiene Validez Concurrente con la Madurez Intelectual.

\section{Validez de Constructo}

El test elaborado constituido por 21 ítems de perspectiva distingue tres planos el frontal, de perfil y horizontal, debiendo confirmarse la presencia de 3 factores diferenciales puesto que la perspectiva de un sujeto no necesariamente debe tener la misma eficiencia para resolver 
problemas según el plano en que se presentan las figuras para algunos individuos pueden tener una mejor estrategia para la reconstrucción mental de planos frontales no igual en los planos de perfil y de repente algo mejor en los planos horizontales y de esta manera los sujetos pueden presentar diversas combinaciones; lo que queremos decir es que la perspectiva diferencia un mínimo de 3 factores que a su vez son parte de la medición de la perspectiva o reconstrucción mental de las figuras.

Para tal fin se analizaron los resultados de los ítems seleccionados mediante el Análisis Factorial por el Método de los Componentes Principales y Rotación Ortogonal Varimax donde se confirmo la existencia de 3 factores que a la luz del contenido nos lleva a afirmar que la solución de los problemas de perspectiva no dependen del plano en que se presenten estos, sino más bien de las dimensiones predominantes en las figuras en la presencia de dos o más elementos de las figuras y en el dominio del volumen en las figuras. Esto significa que en la reorganización mental de los objetos para resolver los problemas de perspectiva cognitiva-mente los sujetos procesan la información a partir de los elementos dominantes estos pueden ser: sólo la forma, la forma más interrelación con otro elemento por ejemplo la figura del semáforo de forma rectangular - circular y en tercer lugar de dominancia el volumen donde la operación mental apunta a la solución del problema a partir del fondo del objeto; concordando con los estudios gestálticos respecto a la percepción, la que ocurre en alternancia entre figura - fondo y la composición de la buena figura.

Tabla $\mathbf{N}^{\circ} 6$

Estudio de la validez de constructo: análisis factorial, rotación varimax de los ítemes del Test de Perspectiva.

\begin{tabular}{|l|l|l|l|}
\hline \multicolumn{1}{|c|}{ Items } & Factor 1 & Factor 2 & Factor 3 \\
\hline Silla & 0.68657 & & \\
Libro & 0.55023 & & \\
Señal & 0.47701 & & \\
Cerca & 0.47172 & & \\
Jarra & 0.43492 & & \\
Botiquín & 0.32958 & & \\
\hline Arco & & 0.62363 & \\
Borrador & & 0.56953 & \\
Archivo & 0.53171 & \\
Tranquera & 0.50672 & \\
Semáforo & 0.50029 & \\
Regla & 0.44556 & \\
\hline Tacho & & 0.61485 \\
Interruptor & & 0.55749 \\
Martillo & & 0.53726 \\
Refrigerador & & 0.39042 \\
Maletín & & 0.33651 \\
\hline \multicolumn{3}{|c|}{ Varianza Total explicada } \\
\hline
\end{tabular}

Los 3 factores hallados explican el $34.2 \%$ de la varianza común y los factores hallados difieren con el supuesto básico que los ítems están agrupados por planos, porque dentro de los factores hallados se encuentran ítems de planos diferentes, como por ejemplo en el Factor 1 la Jarra es un ítem cuya respuesta es el plano frontal mientras que las respuestas en 
los demás ítems de este factor corresponden al plano de perfil.

De lo hallado se confirma que el test elaborado distingue 3 factores que no obedecen a los planos sino más bien a las formas perceptuales que llevan al sujeto a resolver el problema a partir del significado que el sujeto atribuye al objeto, siendo apropiado el termino piagetano de reconstrucción mental de la realidad poniéndose en el lugar del objeto referencia.

\section{COMPARACIONES INTER-GRUPOS}

Para la elaboración de la norma los datos de los 973 sujetos fueron sometidos al análisis de varianza con el objeto de verificar si difieren en función a la edad cronológica, el género y el tipo de colegio de los estudiantes examinados. Los resultados que justifican la elaboración de baremos se reproducen en la siguiente tabla:

Tabla $\mathbf{N}^{\circ} 7$

Análisis de Varianza de los Puntajes del Test de Perspectiva en función a la Edad, el Género y el Tipo de Centro Educativo.

\begin{tabular}{|c|c|c|c|c|c|}
\hline Indice de Variabilidad & SS & Se & $\mathbf{S}^{2} \mathbf{e}$ & $\mathbf{F}$ & $F^{\prime}$ \\
\hline $\begin{array}{l}\text { SS Entre Grupo } \\
\text { SS Dentro Grupo } \\
\text { SS Total }\end{array}$ & \begin{tabular}{|l}
2173.6952 \\
12772.5597 \\
14946.2549
\end{tabular} & $\begin{array}{c}15 \\
957\end{array}$ & $\begin{array}{c}144.91 \\
13.34\end{array}$ & 10.85 & 1.67 \\
\hline $\begin{array}{l}\text { SS Entre Grupo Edad (A) } \\
\text { SS Entre Grupo Genero (B) } \\
\text { SS Entre Grupo Tipo CE (C) }\end{array}$ & $\begin{array}{r}1844.7947 \\
195.7012 \\
570.1802\end{array}$ & $\begin{array}{l}7 \\
1 \\
1\end{array}$ & $\begin{array}{l}263.5421 \\
195.7012 \\
570.1802\end{array}$ & $\begin{array}{l}19.74 \\
14.66 \\
42.74\end{array}$ & $\begin{array}{l}2.01 \\
3.84 \\
3.84\end{array}$ \\
\hline
\end{tabular}

Al concluir el análisis hemos encontrado que:

a) La edad cronológica plantea diferencias significativas y se distinguen dos períodos: el primero de 8 a 11 años y el segundo de 12 a 15 años.

b) El género plantea diferencias significativas.

c) La procedencia en función al Tipo de Centro Educativo Estatal y Particular plantea diferencias significativas.

\section{BAREMOS}

Por lo expuesto queda justificada la elaboración de baremos por período de edad, por género y por tipo de colegio, estos baremos se encuentran en Puntaje T para lo cual los Puntajes Directos se convirtieron a Puntajes Z y estos a su vez a Puntajes T.

\section{Tabla $\mathbf{N}^{\circ} 8$}

Conversión de puntajes directos a puntajes $\mathrm{T}$ de los centros educativos estatales por periodos de edad y por genero

\begin{tabular}{|c|c|c|c|c|}
\hline \multirow[t]{3}{*}{$P D$} & \multicolumn{4}{|c|}{$\begin{array}{c}\text { PUNTUACIONES T } \\
\text { C.E ESTATALES }\end{array}$} \\
\hline & \multicolumn{2}{|c|}{8 a 11 años } & \multicolumn{2}{|c|}{12 a 15 años } \\
\hline & $V$ & $M$ & $V$ & $M$ \\
\hline 21 & 86 & 91 & 72 & 82 \\
\hline 20 & 83 & 87 & 69 & 79 \\
\hline
\end{tabular}




\begin{tabular}{|c|c|c|c|c|}
\hline 19 & 80 & 84 & 67 & 76 \\
18 & 78 & 80 & 65 & 74 \\
17 & 75 & 77 & 62 & 71 \\
16 & 72 & 74 & 60 & 68 \\
\hline 15 & 69 & 70 & 57 & 65 \\
14 & 67 & 67 & 55 & 62 \\
13 & 64 & 64 & 52 & 59 \\
12 & 61 & 60 & 50 & 56 \\
11 & 58 & 57 & 48 & 53 \\
\hline 10 & 56 & 53 & 45 & 50 \\
9 & 53 & 50 & 43 & 47 \\
8 & 50 & 47 & 40 & 44 \\
7 & 47 & 43 & 38 & 41 \\
6 & 44 & 40 & 35 & 38 \\
\hline 5 & 42 & 36 & 33 & 35 \\
4 & 39 & 33 & 31 & 32 \\
3 & 36 & 30 & 28 & 29 \\
2 & 33 & 26 & 26 & 26 \\
1 & 31 & 23 & 23 & 24 \\
0 & 28 & 20 & 21 & 21 \\
\hline
\end{tabular}

Tabla $\mathbf{N}^{\circ} 9$

Conversión de puntajes directos a puntajes $\mathbf{T}$ de los centros educativos particulares por periodos de edad y por género

\begin{tabular}{|c|c|c|c|c|}
\hline \multirow[t]{3}{*}{$P D$} & \multicolumn{4}{|c|}{$\begin{array}{l}\text { PUNTUACIONES T } \\
\text { C.E PARTICULARES }\end{array}$} \\
\hline & \multicolumn{2}{|c|}{8 a 11 años } & \multicolumn{2}{|c|}{$\begin{array}{l}12 \text { a } 15 \\
\text { años }\end{array}$} \\
\hline & $V$ & $M$ & $V$ & $M$ \\
\hline 21 & 77 & & 70 & 73 \\
\hline 20 & 74 & & & 1 \\
\hline 19 & 71 & & & 58 \\
\hline 18 & 69 & & & 56 \\
\hline 17 & 66 & & & 63 \\
\hline 16 & 63 & & 59 & 60 \\
\hline 15 & 61 & & & 58 \\
\hline 14 & 58 & & & 55 \\
\hline 13 & 55 & & & 53 \\
\hline 12 & 53 & & & 50 \\
\hline 11 & 50 & & & 47 \\
\hline 10 & 47 & & & 45 \\
\hline 9 & 45 & & & 42 \\
\hline 8 & 42 & & & 40 \\
\hline 7 & 39 & & & 37 \\
\hline 6 & 37 & & & 34 \\
\hline 5 & 3 & & & 32 \\
\hline 4 & 31 & & & 29 \\
\hline 3 & 29 & & & 27 \\
\hline 2 & 26 & & & 24 \\
\hline 1 & 23 & 25 & 25 & 21 \\
\hline & 21 & 22 & 23 & 19 \\
\hline
\end{tabular}


A continuación se reproduce el baremo por categorías para lo cual los puntajes directos se convirtieron a puntajes T:

Tabla $\mathbf{N}^{\circ} \mathbf{1 0}$

Conversión de Puntajes T a Categorías de Rendimiento en el Test de Perspectiva en una muestra de 973 estudiantes.

\begin{tabular}{|c|c|c|}
\hline Puntajes & Categorías & No. Alumnos \\
\hline $71-80$ & Muy Superior & 25 \\
$61-70$ & Superior & 123 \\
$56-60$ & Normal Alto & $1 I 8$ \\
$46-55$ & Normal & 343 \\
$41-45$ & Normal Bajo & 185 \\
$31-40$ & Bajo & 173 \\
$20-30$ & Muy Bajo & 6 \\
\hline \multicolumn{2}{|c|}{ Total } & 973 \\
\hline
\end{tabular}

\section{CONCLUSIONES}

1. Fueron 17 los Ítems seleccionados que presentan Índices de dificultad entre 0.40 a 0.60 y coeficientes de discriminación tetracórica significativos al 0.05 ; esto significa que el $81 \%$ de los Ítems están dentro de los criterios esperados, mientras que el 19\% están descartados porque no cumplen los requisitos antes señalados cuyo valor Ji Cuadrado de 38.44 resulta ser muy significativo aún para el 0.01 , por lo que se concluye que el número de ítems aceptados del Test de Perspectiva son significativos.

2. El test elaborado es confiable por el Método de Consistencia Interna al encontrar coeficientes mayores a 0.80 según el Tipo de Colegio y la Muestra Total, esto significa que el test es preciso al medir la perspectiva. Por el Método de las Mitades la prueba tiene una alta confiabilidad ya que sus errores de medición van desde un mínimo de 0.05 hasta un máximo de 0.08 .

3. El test resultó tener validez concurrente al correlacionar los puntajes totales del Test de Perspectiva con la Madurez Intelectual de la Batería de Aptitudes Diferenciales y Generales - Nivel Elemental (B.A.D.Y.G-E), encontrando coeficientes significativos al 0.05 de margen de error; la validez de constructo diferenció 3 factores que no son las vistas o planos que fueron explorados, es por ello que estos nuevos factores deben ser investigados.

4. Los factores discriminados por la validez de constructo están en función a las dimensiones predominantes en las figuras, en la presencia de dos o más elementos de las figuras y en el dominio del volumen en las figuras, según estas características se deben realizar investigaciones para confirmar si este es el principio que agrupa a los factores.

5. Respecto a la edad se obtuvieron Índices de variabilidad significativos y además se llegaron a distinguir dos períodos: el primero de 8 a 11 años y el segundo de 12 a 15 años siendo períodos similares a los propuestos por Piaget.

6. Según los resultados obtenidos en la diferencia de medias y la prueba $\mathrm{S}$ podemos afirmar que los varones presentan un mayor rendimiento en el Test de Perspectiva que las mujeres.

7. Al transformar los puntajes directos a puntuaciones $\mathrm{T}$ podemos observar que los estudiantes pertenecientes a Centros Educativos Particulares alcanzan un mayor rendimiento en el Test de Perspectiva que los estudiantes de Centros Educativos Estatales. 


\section{BIBLIOGRAFIA}

Beard R. (1971) Psicología Evolutiva de Piaget, Buenos Aires: Editorial Kapeluz.

Bee H. (1987) El Desarrollo de la Persona, México: Editorial Hada.

Forgus R. - Melamed L. (1989) Percepción, México: Editorial Trillas.

Fraisse P. - Piaget J. (1973) La Percepción, Buenos Aires: Editorial Paidos.

García Gonzáles E. (1995) Piaget, México Editorial Trillas.

Holloway, G.E.T. (1982) La Concepción del Espacio en el Niño según Piaget, Barcelona:

Ediciones Paidos. Ira reimpresión.

Piaget J. - Inhelder B. (1981) Psicología del Niño, Madrid: Ediciones Morata.

Phillips, J. L. Jr. (1984) Los Orígenes del Intelecto según Piaget, Barcelona: Editorial Fontanella. 